\title{
Classical Trajectory Calculations of Infrared Multiphoton Absorption and Dissociation
}

\author{
S. K. GRAY
}

The James Franck Institute, The University of Chicago, 5640 Ellis Avenue, Chicago, Illinois 60657

\section{J. R. STINE}

M-1 MS-C920, Los Alamos National Laboratory, Los Alamos, New Mexico 87545, USA

D. W. NOID $\dagger$

Department of Chemistry, Oak Ridge National Laboratory, Oak Ridge, Tennessee 37831, USA and Department of Chemistry, University of Tennessee, Knoxville, Tennessee 37996-1600, USA

(Received December 13, 1984)

A review of classical trajectory calculations of infrared multiphoton absorption and dissociation is presented. These calculations have demonstrated that the classical method can be useful for interpreting experimental results and for understanding the fundamental processes by which molecules absorb photons from the laser.

\section{INTRODUCTION}

An understanding of how molecules interact with intense laser radiation is crucial to the design and interpretation of experiments on multiphoton excitation and dissociation, ${ }^{1}$ and photoselective chemistry. ${ }^{2}$ In this paper, we present an overview of how classical methods have contributed to this understanding.

\footnotetext{
$\dagger$ Research sponsored by the U.S. Department of Energy under contract DE-AC05840R21400 with Martin Marietta Energy Systems, Inc.
} 
Of course, the use of classical dynamics to describe molecular energy absorption is only an approximation, and the correct approach is via quantum dynamics. Indeed, significant advances have been made recently in applying quantum mechanics to laser-molecule problems, ${ }^{3}$ and model systems with as many as 15000 molecular states interacting with a laser mode have been studied. ${ }^{3 \mathrm{c}}$ However, most realistic molecular systems have such high densities of states (e.g., in $\mathrm{SF}_{6}$ the density of states is $\sim 10^{6}$ states $/ \mathrm{cm}^{-1}$ at an energy of $\sim 10000 \mathrm{~cm}^{-1}$ ) that a full quantum approach, especially if dissociation (continuum states) is to be considered, is still out of the question. Fortunately, it is in the limit of intense laser fields and high densities of states that classical dynamics is most valid.

For the past three decades, classical mechanics (classical trajectories) has been used as a tool to quantitatively understand the chemistry in scattering systems. ${ }^{4}$ For those cases where exact quantum mechanical calculations could be performed, the classical approximation was found to be reasonable for the calculation of various scattering properties (e.g., scattering cross sections and rate constants) and failed only when classically forbidden processes were dominant, such as tunneling or interference effects. In most cases, the latter phenomena were adequately treated using semiclassical methods.

The last decade has seen the use of classical mechanical methods to describe the dynamics of multidimensional anharmonic unimolecular systems. ${ }^{5}$ These methods have led to new concepts related to random and nonrandom energy transfer and the breakdown of the conventional normal mode and perturbative analyses for vibrationally excited molecules.

The use of classical mechanics to describe these systems has proved popular because, even for modest systems, quantum mechanical methods require extraordinary amounts of computational time and storage. In general, the complexity of the quantum mechanical problem varies as some power of the number of degrees of freedom, whereas the classical complexity varies only linearly. Probably more importantly, the classical method allows a visualization of the dynamics of the process that is not possible or is obscured in the quantum calculations.

For example, classical methods have demonstrated the existence of two very different types of motion in bound systems. ${ }^{5}$ One type, a very regular motion, termed quasiperiodic, and another type, a very 
irregular motion, termed chaotic, have been useful in characterizing the energy flows in polyatomic systems.

Because a classical Hamiltonian can be written for the interaction of intense laser radiation with a molecule, it is of interest to apply these classical methods to such systems. The laser-molecule system is of special theoretical and experimental interest because, to a certain extent, the coupling between the laser and the molecule can be altered by changing the laser power or frequency. In this way, certain processes such as molecular dissociation can be enhanced by simply scanning the laser frequency.

In this paper, we hope to demonstrate how classical mechanics has been used as a tool to investigate realistic systems and also how it has contributed to our understanding of the laser-molecule interaction that perhaps could not be obtained by using other methods. In Section II, the classical mechanical method is outlined, and in Section III, some of the results obtained using these methods are reviewed. A discussion of these results and areas for future investigations are presented in Section IV.

\section{CLASSICAL TRAJECTORY METHODS}

The classical Hamiltonian describing the interaction of a molecule with a laser can be written as

$$
H(\mathbf{p}, \mathbf{x}, t)=H_{\mathrm{mol}}(\mathbf{p}, \mathbf{x})-\varepsilon \cdot \boldsymbol{\mu}(\mathbf{x}) \cos (\omega t)
$$

where $\mathbf{x}$ is the nuclear coordinate vector, $\mathbf{p}$ is the conjugate momentum vector, $\boldsymbol{\mu}$ is the dipole moment vector of the molecule, $\boldsymbol{\varepsilon}$ is the time independent part of the electric field vector of the laser, and $\omega$ is its frequency. $H_{\text {mol }}$ is the Hamiltonian for the isolated molecule and is of the form

$$
H_{\text {mol }}(\mathbf{p}, \mathbf{x})=\mathbf{p} \cdot \mathbf{p} / 2 m+V(\mathbf{x})
$$

where $m$ is the reduced mass of the molecule and $V(\mathbf{x})$ is the potential energy function.

Note that Eq. (1) differs from the usual Hamiltonians used in studying molecular collisions in that it is explicitly time-dependent, so that energy is not a constant of the motion. This, of course, is neither a problem in principle nor in practice, but it is sometimes useful to work within a time-independent Hamiltonian. It is always 
possible to convert a time-dependent to a time-independent Hamiltonian by introduction of an extended phase space, and this has been demonstrated explicitly for the laser-molecule problem by Stine and Noid. ${ }^{6}$ Another point about the Hamiltonian given by Eq. (1) is that it does not allow for certain (low intensity) effects such as spontaneous emission. Although popular folklore is that such effects are purely quantum mechanical, Miller ${ }^{7}$ has shown with clarity that a classical analogue of the quantized field Hamiltonian can be constructed that does indeed include spontaneous emission. (See also the comment on this by Milonni ${ }^{8}$ and Miller's reply. $\left.{ }^{9}\right)$ Miller's Hamiltonian is also time independent and is not much more difficult to use than Eq. (1). Indeed, it has been used in classical studies of a Morse oscillator in an intense laser ${ }^{10}$ and also certain laser-induced chemical reactions. ${ }^{11}$ However, since the Miller Hamiltonian reduces for all practical purposes to Eq. (1) in the limit of the high field strength studies to be reviewed here, we will not discuss it any further, except to note that recently Shirts and Davis ${ }^{12}$ showed that Miller's Hamiltonian is also derivable from classical electrodynamics.

Typically, an ensemble of $N$ initial values of the molecular position and momentum variables $\left(\mathbf{x}_{0}, \mathbf{p}_{0}\right)$ is selected consistent with some distribution $\rho\left(\mathbf{x}_{0}, \mathbf{p}_{0}\right)$ which represents the state of the molecule "before" the laser is turned on. For example, $\rho\left(\mathbf{x}_{0}, \mathbf{p}_{0}\right)$ might be a microcanonical distribution corresponding to a particular energy level of the isolated molecule. It is occasionally desired, in addition to averaging over the molecular phases, to average over the laser phase as well (for example, in studies of molecules interacting with multiple lasers). This is accomplished by replacing $\cos (\omega t)$ in Eq. (1) with $\cos$ $(\omega t+\delta)$ and choosing $\delta$ randomly in the interval $(0,2 \pi)$ for each trajectory.

Once the initial conditions are selected, Hamilton's equations

$$
\begin{aligned}
& \dot{\mathbf{x}}=\partial \mathbf{H} / \partial \mathbf{p} \\
& \dot{\mathbf{p}}=-\partial \mathbf{H} / \partial \mathbf{x}
\end{aligned}
$$

are integrated to produce a trajectory $(\mathbf{x}(t), \mathbf{p}(t))$.

One quantity of interest is the time-dependent energy absorption by the molecule, $E_{\text {mol }}(t)$, given by a simple average over the ensemble

$$
E_{\mathrm{mol}}(t)=\frac{1}{N} \sum_{k=1}^{N} H_{\mathrm{mol}}(\mathbf{p}(t), \mathbf{x}(t))
$$


This quantity gives a qualitative indication of the rate at which the molecule is absorbing energy from the field and the degree of excitation of the molecule after interacting with the laser for a time $t$.

In all of the classical studies thus far, the laser mode contains a sufficient number of photons to dissociate the molecule. However, because of the nature of the coupling between the molecule and the laser, the rate at which this energy is transferred can be extremely slow. To compensate for this phenomenon, the classical calculations require larger laser fields to see an effect on a picosecond time scale than may be required experimentally.

\section{CLASSICAL STUDIES OF MOLECULAR EXCITATION AND DISSOCIATION}

One early classical study was of multiphoton absorption in $\mathrm{SF}_{6}$ by Poppe. ${ }^{13}$ His molecular potential energy and dipole moment functions reproduced the spectroscopic properties of $\mathrm{SF}_{6}$, and he included all of the rotational and vibrational degrees of freedom in his calculations. He looked at the amount of energy absorbed by the molecule at various exciting frequencies of the laser and observed that the mean energy absorption depends on the fluence (i.e., energy density or timeintegrated intensity) and not on the intensity of the laser. Indeed, his results on the mean energy absorption agree reasonably well with experimental results both in terms of fluence and in terms of laser frequency dependence. Unfortunately, Poppe was unable, for computational reasons, to compute the threshold fluence for dissociation directly, but resorted to statistical arguments based on his calculated energy distributions. Nonetheless, his estimated fluence threshold of about $1 \mathrm{~J} / \mathrm{cm}^{2}$ compares favorably with the experimental value of $1.4 \mathrm{~J} / \mathrm{cm}^{2}$. However, Poppe's dissociation probability rose much more steeply with increasing fluence than the experimental results, which as he notes is probably caused by the statistical approximation rather than by the actual trajectory data.

One of the earliest classical trajectory studies of multiphoton dissociation of a polyatomic system was by Noid et al. ${ }^{14}$ on $\mathrm{CD}_{3} \mathrm{Cl}$. Although features of the potential energy and dipole moment used in this study were idealized (e.g., the potential energy surface only allowed for $\mathrm{C}-\mathrm{Cl}$ bond rupture and the dipole moment function was only 
dependent on the $\mathrm{C}-\mathrm{Cl}$ bond distance), all of the vibrational and rotational degrees of freedom were included. A number of experimental findings on multiphoton absorption and dissociation were qualitatively verified in this study. For example, an energy fluence dependence for multiphoton dissociation was noted and, with the possible exception of extremely intense, short laser pulses, energy randomization and statistical (RRKM) product translational energy distributions appeared unavoidable.

The results of Noid et al. ${ }^{14}$ are therefore consistent with the fact that mode specific multiphoton excitation and dissociation is very difficult to achieve. ${ }^{1}$ The usual qualitative quantum mechanical explanation of this fact is that the energy levels close to dissociation that are ultimately reached after the absorption of many photons lie in a "quasicontinuum" where there is sufficient mixing between zero-order modes to effectively delocalize the excitation in a statistical manner about the molecule. ${ }^{1,15}$ The classical analogue of this behavior, i.e., what must be operative in the study of Noid et al., involves substantial energy flow between the classical degree of freedom. It is then intriguing to speculate on the role of classical chaos in the multiphoton absorption and dissociation process, since in the case of isolated molecular systems trajectories that behave in a chaotic fashion are known to lead to energy randomization. ${ }^{5}$

Similar conclusions to those of Noid et al. ${ }^{14}$ were reached by Hansel $^{16}$ in a classical study of the multiphoton dissociation of a model two degrees of freedom system. Hansel's "molecule" consisted of a Morse oscillator coupled to a harmonic oscillator. The simplicity of this model allowed for a more thorough analysis than that of Noid et al. ${ }^{14}$ For example, Hansel was able to locate an approximate threshold intensity for significant multiphoton dissociation of his system, $\sim 6 \mathrm{GW} / \mathrm{cm}^{2}$, and also carried out a systematic study of the dependence of the dissociation probability on frequency. He found that multiphoton dissociation occurred over a very broad range (FWHM $\sim 250 \mathrm{~cm}^{-1}$ for $I=36 \mathrm{GW} / \mathrm{cm}^{2}$ ) reflecting the strong coupling (to be discussed further below) inherent in his system but probably typical of real molecular systems. Hansel, as did Noid et al., ${ }^{14}$ used the concept of the laser inducing a "transition" from quasiperiodic to chaotic behavior in individual trajectories. Actually, this sort of analysis is only qualitative since by ordinary criteria of nonlinear dynamics, ${ }^{5}$ such a trajectory would be classified as chaotic. That is, although the 
laser degree of freedom has a very large energy, the rate at which this energy flows into the molecule is very slow, and hence the molecule may appear quasiperiodic on short time scales but eventually executes irregular motion. A simple visualization of this phenomenon is shown qualitatively by plotting the energy in the molecule, $E_{\mathrm{mol}}$, as a function of time. For example, this was demonstrated in one of the first polyatomic systems to be studied, $\mathrm{CD}_{3} \mathrm{Cl}$. Here a potential energy surface that allowed for dissociation of the $\mathrm{C}-\mathrm{Cl}$ bond was used, and the laser had a frequency close to the $1361 \mathrm{~cm}^{-1}$ fundamental frequency of $\mathrm{CD}_{3} \mathrm{Cl}$. Figure 1 shows the energy in the molecule as a function of time. It is seen that the molecule seems to execute a regular type of motion up to $40 \mathrm{ps}$ (the classical analogue of Rabi flops) and then rapidly absorbs energy and executes an irregular type of motion, and then later goes to dissociation.

The concept of the laser making a molecular system"go stochastic" is commonly invoked but still is not very well understood.

Another system with two degrees of freedom exhibiting similar behavior was studied by Ramaswamy, Siders and Marcus. ${ }^{17}$ Hansel also studied a three degree of freedom model for ozone multiphoton dissociation, but only gave a brief account of his results. ${ }^{18}$

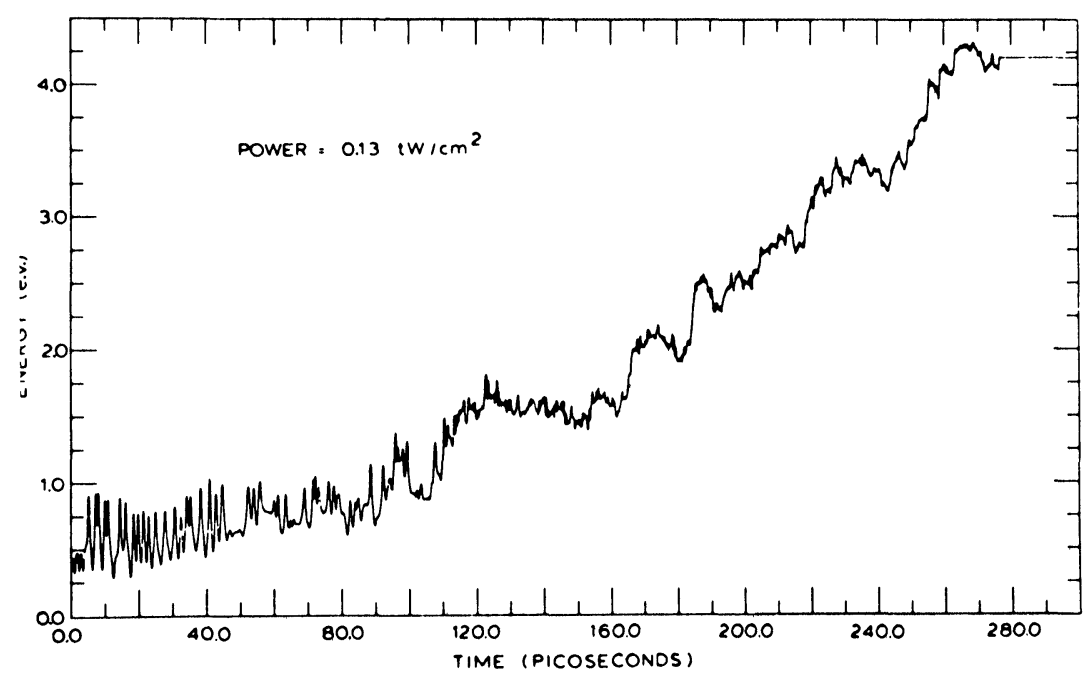

FIGURE 1 Internal energy of a single molecule of $\mathrm{CD}_{3} \mathrm{Cl}$ in a laser field. 
Hansel's original system was studied in more detail by Martin and Wyatt, ${ }^{19}$ who noted various trajectory types. For example, they noted qualitatively four different types of dissociating trajectories and two types of nondissociating trajectories. Although the relative fraction of trajectory types varied strongly with laser frequency, they noted for the laser frequency leading to optimal dissociation that most trajectories were of "type I" or "type II", both of these types displaying to varying degrees the regular to chaotic behavior already discussed. Classical chaos was also found to be important for all four types of dissociating trajectory. Martin and Wyatt also analyzed product translational and vibrational distributions, which were found to agree reasonably well with RRKM predictions: their translational energy distribution was well fit by RRKM theory and there was equipartitioning of energy between vibration and translation.

Davis, Wyatt and Leforestier ${ }^{20}$ studied further the properties of Hansel's original system and variations of it. They noted Hansel's original system was Fermi resonant, i.e., that the ratio of normal mode frequencies was $2: 1$, which would imply strong mode-mode energy transfer. Indeed, Hansel's isolated molecular system exhibits much classical chaos for energies as low as half the dissociation energy. They showed the importance of vibrational energy transfer in leading to multiphoton dissociation by showing that very little dissociation takes place when no coupling is allowed between the modes. They also showed that when coupling is included, multiphoton dissociation can result even when the non-dissociative mode is pumped; i.e., fast vibrational energy transfer to the dissociative mode can occur. Another interesting aspect of their investigations was the nature of the product distributions. For example, by increasing the laser intensity, it was possible for the laser pumping to compete against the vibrational energy transfer allowing for more energy to be effectively trapped in the nondissociative bond and, ultimately, increased vibrational excitation in the products. Increased product excitation was also seen to occur in systems with less coupling between modes.

Because of the availability of highly accurate potential energy and dipole moment functions for diatomic molecules, the hydrogen fluoride-laser system has proved popular. This system also has the advantage that quantum mechanical calculations are also feasible, making for direct comparison with the classical calculations. Also, a diatomic molecule serves as a model isolated vibrational mode in a 
polyatomic system so that its properties contribute in a qualitative sense to the understanding of molecular laser systems in general. Therefore, much work has been done on the diatomic-laser problem, and this is now discussed.

Walker and Preston ${ }^{21}$ were the first to present a classical/quantum comparison of the dynamics of a model nonrotating HF molecule in a strong laser. A Morse oscillator was used to describe the diatomic molecule, and the linear dipole moment approximation was made. The laser intensities used in this study ranged between $11.1 \mathrm{TW} / \mathrm{cm}^{2}$ $\left(A_{0}=0.15 \mathrm{eV} / a_{0}\right.$ in Walker and Preston's variables and units) and $44.4 \mathrm{TW} / \mathrm{cm}^{2}\left(A_{0}=0.30 \mathrm{eV} / a_{0}\right)$, and most of the pulse lengths were 31 optical cycles $(\sim 0.3 \mathrm{ps})$, although results for $100(\sim 0.8 \mathrm{ps})$ and 960 $(\sim 8 \mathrm{ps})$ optical cycles were also presented. Very good agreement between the classical and quantum predictions of the pulse time averaged energy absorption as a function of laser frequency was noted, except at laser frequencies corresponding to multiphoton resonances where classical mechanics did not exhibit the same pronounced effects as quantum mechanics.

Dardi and $\mathrm{Gray}^{22}$ went on to study Walker and Preston's system at much lower intensities of $\sim 1 \mathrm{TW} / \mathrm{cm}^{2}$ and noted the classical/quantum discrepancies, particularly as the multiphoton resonances became more pronounced. Dardi and Gray also considered the case when rotations were included and remarked that rotational fine structure (e.g., selection rules) is completely absent in the classical picture. However, rotationally (and time) averaged classical and quantum probabilities were in better accord.

A diatomic, however, with very few states (HF has only 23 vibrational states) is a very extreme case for comparing classical and quantum mechanics, and it is encouraging that the level of agreement noted above is so good! The discrepancy of the classical/quantum results for the multiphoton resonances deserves further comment, however. It is our opinion that classical mechanics "misses" multiphoton resonances in the case of diatomic molecules because, for the intensities studied above, the motion is still very regular-i.e., chaos plays a relatively insignificant role in the above diatomic studies. Davis and Wyatt ${ }^{10}$ were the first to plot out Poincare surfaces of section of the classical trajectories for the nonrotating HF problem which shows this degree of regularity. These surfaces of section were generalized to a two-laser system by Stine and Noid. ${ }^{6}$ Davis and Wyatt ${ }^{10}$ also 
noted that certain multiphoton transitions were forbidden classically and may be due (e.g., semiclassically) to a tunneling phenomenon known as dynamical tunneling. Gray, ${ }^{23}$ using a previously developed classical nonlinear resonance model for a Morse oscillator interacting with a laser, ${ }^{24}$ noted that for sufficiently small laser intensities, dynamical tunneling may be significant in overtone and fundamental absorption as well. Indeed, ordinary (low intensity) spectroscopy is due entirely to dynamical tunneling from a semiclassical point of view. Also, the initial steps in the multiphoton absorption process before the quasicontinium is reached may well be due to such effects. Conversely, as intensity is increased, the effects of dynamical tunneling should diminish and a purely classical picture of absorption should suffice. The effect of dynamical tunneling can also be diminished by increasing the number of vibrational degrees of freedom, thus allowing for an increased role of classical chaos in achieving absorption. For example, very reasonable agreement between classical and quantum energy absorption spectra in a model triatomic system has been noted. ${ }^{25}$ For these reasons, we believe that while one should be somewhat cautious about classical studies omitting such quantum effects, the classical model of multiphoton absorption and dissociation of polyatomics in intense lasers is probably reasonable, particularly with regard to behavior in the quasicontinium energy regime.

Perhaps more relevant experimentally are the studies by Stine and Noid ${ }^{26,27}$ that have showed the effect of employing two lasers to excite a diatomic system. (The two-laser Hamiltonian is obtained from Eq. (1) simply by adding the term $-\varepsilon \cdot \mu(\mathbf{x}) \cos \omega_{2} t$. Stine and Noid first considered a model (nonrotating) 7-vibrational state Morse oscillator interacting with one and two lasers. They found that the fraction of dissociating trajectories could be doubled by employing a relatively weak laser near the fundamental resonance, and another stronger laser with a frequency significantly red-shifted. Typical dissociating trajectories exhibited behavior analogous to Figure 1. They also considered the case of both lasers being the same strength and noted that the threshold intensity for dissociation was 10 times smaller than in the one-laser case. Analogous calculations with the Morse oscillator corresponding to a nonrotating HF molecule showed that the threshold for dissociation with two lasers (and equal field strengths) was $\sim 13 \mathrm{TW} / \mathrm{cm}^{2}$ compared to $\sim 125 \mathrm{TW} / \mathrm{cm}^{2}$ in the one-laser case. The time scale for dissociation was in the picosecond range. The introduc- 
tion of a second laser, as noted by Stine and Noid, has the effect of increasing the amount of classical chaos and can be interpreted via classical nonlinear resonance theory. While no diatomic molecule has been multiphoton dissociated in the lab yet, a number of experiments on polyatomic systems have illustrated this two-laser effect. ${ }^{28}$ The earlier classical studies on $\mathrm{CD}_{3} \mathrm{Cl}$ by Noid et al. ${ }^{14}$ and classical and quantum studies of a model triatomic by Noid, Bottcher and Koszykowski ${ }^{25}$ also confirm the two-laser effect.

In considering a rotating and vibrating HF molecule, Stine and Noid $^{29,30}$ also showed classically that the relative polarization of the two lasers also matters: a parallel arrangement of laser polarizations leads to twice as much energy absorption than a perpendicular arrangement. Finally, Noid and Stine ${ }^{31}$ also demonstrated that use of a static electric field and a single laser tuned near the fundamental could also increase energy absorption by diatomic molecules, and their results, while pertaining to $\mathrm{HF}$, were in qualitative accord with experiments on $\mathrm{CF}_{2} \mathrm{HCl}^{32}$

We now consider a number of classical studies, mostly on the diatomic problem, that sought to find out how best to excite the system. Christoffel and Bowman ${ }^{33}$ looked at the nonrotating HF problem but considered both the effects of initial vibrational state and nonlinear vs. linear dipole function. For the case of the laser tuned slightly off the fundamental $\nu=0 \rightarrow 1$ resonance, they found, in addition to significant absorption from the initial $\nu=0$ state, significant absorption from $\nu \geqslant 9$. They interpreted the latter observation to be due to the onset of significant overtone absorption. It is important to note that a subsequent quantum study by Dardi and $\mathrm{Gray}^{34}$ showed essentially the same behavior. Davis and Wyatt ${ }^{10}$ analyzed these classical trends in terms of classical resonances, noting that in the region of overtone absorption a $3: 2$ resonance between molecular vibration frequency and laser frequency exists, as well a significant chaotic motion, which probably leads to the increased energy absorption (see also $\mathrm{Gray}^{24}$ ). Dardi and Gray ${ }^{34}$ discuss this point further. Finally, Christoffel and Bowman also considered $\nu=0$ molecules with an intense $\left(44 \mathrm{TW} / \mathrm{cm}^{2}\right)$ laser tuned exactly to the $\nu=0 \rightarrow 2$ overtone frequency and noted that with a linear dipole moment, no classical absorption would take place, whereas with a nonlinear dipole moment, overtone adsorption would indeed occur. Actually, Gray ${ }^{23}$ and Dardi and Gray ${ }^{34}$ showed, using quantum mechanics, very significant overtone absorption takes place 
even with the linear dipole. The absence of the absorption in classical mechanics is due semiclassically to the dynamical tunneling effect discussed earlier. ${ }^{23}$ Dardi and $\mathrm{Gray}^{34}$ did show that the nonlinear dipole would lead to a slightly broader energy absorption spectrum, but the overall effect of a nonlinear dipole function is less significant in quantum mechanics.

\section{CONCLUDING REMARKS}

It has been demonstrated that classical mechanics complements statistical theories and experimental methods in understanding multiphoton absorption and dissociation.

Numerous investigations on both model and realistic systems have demonstrated the energy randomization in the molecule once the energy absorption reaches a certain threshold. These observations confirm the difficulty of experimental efforts in achieving mode specific chemistry.

The ability to distinguish between regular and irregular motions using classical methods helps in predicting where classical and quantum results might differ. For example, it is probable that the classical calculations "miss" the multiphoton resonances in the case of diatomic molecules if the motion is still very regular. Further, this ability to distinguish between the quasiperiodic and stochastic regimes plays a crucial role in deciding if and when statistical methods are valid.

Finally, we remark that alternative classical calculations-for example, the recent work of Ackerholt, Galbraith and Milonni ${ }^{35}$ that was based on approximating with classical dynamics the time dependence of the laser-molecule system's creation and annihilation operators- also point to the importance of chaos in the multiphoton absorption process.

\section{Acknowledgement}

This paper was written when one of us (DWN) was a Visiting Associate Professor to the Department of Chemistry, University of Wisconsin. We (SKG, JRS, and DWN) wish to acknowledge support for this collaboration from NATO (Grant 229.81). 


\section{References}

1. P. A. Schulz, Aa. S. Sudbø, D. J. Krajnovich, H. S. Kowk, Y. R. Shen and Y. T. Lee, Ann. Rev. Phys. Chem. 30, 379 (1979).

2. See articles in Adv. Chem. Phys. 47 (1981).

3. (a) R. E. Wyatt, G. Hase and H. S. Taylor, Phys. Rev. A 28, 815 (1983); (b) M. Quack and E. Stutcliffe, Chem. Phys. Lett. 105, 147 (1984); (c) A. Nauts and R. E. Wyatt, Phys. Rev. Lett. 51, 2238 (1983).

4. (a) R. N. Porter, Ann. Rev. Phys. Chem. 25, 317 (1974); (b) R. N. Porter and L. M. Raff, in: Dynamics of Molecular Collisions, Part B, ed. W. H. Miller (Plenum, 1976), p. 1.

5. D. W. Noid, M. L. Koszykowski and R. A. Marcus, Ann. Rev. Phys. Chem. 32, 267 (1981).

6. J. R. Stine and D. W. Noid, J. Phys. Chem. 86, 3735 (1982).

7. W. H. Miller, J. Chem. Phys. 69, 2188 (1978).

8. P. W. Milonni, J. Chem. Phys. 72, 787 (1980).

9. W. H. Miller, J. Chem. Phys. 72, 783 (1980).

10. M. J. Davis and R. E. Wyatt, Chem. Phys. Lett. 86, 235 (1982).

11. A. E. Orel and W. H. Miller, 70, 4393 (1979).

12. R. B. Shirts and T. F. Davis, J. Phys. Chem. 88, 4665 (1984).

13. D. Poppe, Chem. Phys. 45, 371 (1980).

14. D. W. Noid, M. L. Koszykowski,.R. A. Marcus and J. D. McDonald, Chem. Phys. Lett. 51, 540 (1977).

15. N. Bloembergen and E. Yablonovitch, Phys. Today 31, 23 (1978).

16. K. D. Hansel, Chem. Phys. Lett. 57, 619 (1978).

17. R. Ramaswamy, P. Siders and R. A. Marcus, J. Chem. Phys. 78, 4418 (1981).

18. K. D. Hansel, in: Laser Induced Processes in Molecules, eds. K. L. Kompe and S. D. Smith (Springer, 1979), pp. 145-148.

19. D. L. Martin and R. E. Wyatt, Chem. Phys. 64, 203 (1982).

20. M. J. Davis, R. E. Wyatt and C. Leforestier, in: Intramolecular Dynamics, eds. J. Jortner and B. Pullman (Reidel, 1982), pp. 403-427.

21. R. B. Walker and R. K. Preston, J. Chem. Phys. 67, 2017 (1977).

22. P. S. Dardi and S. K. Gray, J. Chem. Phys. 77, 1345 (1982).

23. S. K. Gray, Chem. Phys. 83, 125 (1984).

24. S. K. Gray, Chem. Phys. 75, 67 (1983).

25. D W. Noid, C. Bottcher and M. L. Koszykowski, Chem. Phys. Lett. 72,397 (1980).

26. J. R. Stine and D. W. Noid, Opt. Commun. 31, 161 (1979).

27. D. W. Noid and J. R. Stine, Chem. Phys. Lett. 65, 153 (1979).

28. e.g., R. V. Ambartzumian, Y. A. Brokhov, V. S. Letokhov, G. N. Marakov, A. A. Puretzkii and N. P. Furzikov, JETP Letters 23, 194 (1976).

29. J. R. Stine and D. W. Noid, Chem. Phys. Lett. 77, 287 (1981).

30. D. W. Noid and J. R. Stine, Opt. Commun. 37, 187 (1981).

31. D. W. Noid and J. R. Stine, J. Chem. Phys. 76, 4947 (1982).

32. P. Gozel and It. van den Bergh, J. Chem. Phys. 74, 1724 (1981).

33. K. M. Christoffel and J. M. Bowman, J. Phys. Chem. 85, 2159 (1981).

34. P. S. Dardi and S. K. Gray, J. Chem. Phys. 80, 4738 (1984).

35. J. R. Ackerholt, H. W. Galbraith and P. W. Milonni, Phys. Rev. Lett. 51, 1259 (1983). 